\title{
ON RECONSTRUCTING A GRAPH
}

\section{ROBERT L. HEMMINGER}

1. Introduction. The term "graph" will here denote an unoriented finite graph without loops or multiple edges. $V(G)$ will denote the vertex set of $G$ and $E(G)$ will denote the edge set. If $a \in V(G)$, we will let $G_{a}$ denote the graph obtained from $G$ by deleting the vertex $a$ and the edges adjacent to $a$. If $e \in E(G)$ we will let $G^{e}$ denote the graph obtained from $G$ by deleting $e$. P. J. Kelly [3] has proven the following theorem: If $G$ and $H$ are trees and $\sigma: V(G) \rightarrow V(H)$ is a 1-1 onto function such that $G_{a} \cong H_{\sigma(a)}$ for all $a \in V(G)$, then $G \cong H$. He conjectures that this theorem is true for arbitrary graphs and has verified it for graphs on $n$ vertices where $2<n \leqq 6$. An equivalent formulation of Kelly's conjecture is as follows: $G$ is uniquely determined, up to isomorphism, by the collection $\left\{G_{a}\right\}_{a \in V(G)}$. We will refer to this as the vertex problem. If a graph $G$ is uniquely determined, up to isomorphism, by a given collection of subgraphs we will say that $G$ can be reconstructed from that collection of subgraphs. It needs to be emphasized that the given subgraphs have no labellings.

Harary and Palmer [1] generalized Kelly's theorem on trees by showing that a tree $G$ can be reconstructed from the $G_{a}$ with $a$ of degree one in $G$.

In [2], Harary asks if $G$ can be reconstructed from the collection $\left\{G^{e}\right\}_{e \in E(G)}$. We will refer to this as the edge problem. The purpose of this paper is to show that the edge problem is a special case of the vertex problem.

Undefined terms in the paper can be found in the above-mentioned papers or in [4].

2. The use of the line graph. If $G$ is a graph, then the line graph of $G$, denoted by $L(G)$, is the graph with $V(L(G))=E(G)$ and with $\left(e_{1}, e_{2}\right) \in E(L(G))$ if and only if $e_{1}$ and $e_{2}$ are adjacent in $G$.

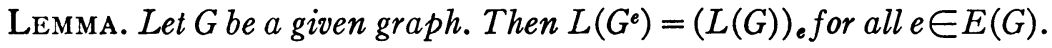

Proof. Both graphs have $E(G)-\{e\}$ as vertex set, and if $e_{1}$, $e_{2} \in E(G)-\{e\}$, then the criterion for $\left(e_{1}, e_{2}\right)$ to be an edge in either graph is the same; namely that $e_{1}$ and $e_{2}$ be adjacent in $G$.

Since the number of isolated vertices in $G$ can be discovered from the $\left\{G^{l}\right\}_{e \in E(G)}$ we assume in the following that $G$ and $H$ have no isolated vertices.

Received by the editors October 4, 1967. 
THEOREM. The edge problem is equivalent to the vertex problem for line graphs; i.e., a solution to the edge problem would give a solution to the vertex problem for line graphs and conversely.

Proof. Suppose the vertex problem is true for line graphs. Let $G$ and $H$ be graphs and let $\tau: E(G) \rightarrow E(H)$ be a 1-1 onto function such that $G^{e} \cong H^{\tau(e)}$ for all $e \in E(G)$. By the Lemma we then have $(L(G)$ )e $=L\left(G^{e}\right) \cong L\left(H^{\tau(e)}\right)=(L(H))_{\tau(e)}$ for all $e \in E(G)$. But then $\tau: V(L(G))$ $\rightarrow V(L(H))$ is a 1-1 onto function such that $(L(G))_{e} \cong(L(H))_{\tau(e)}$ for all $e \in V(L(G)$ ) so by our assumption $L(G) \cong L(H)$. Now $G$ and $L(G)$ have the same number of components so $G$ and $H$ have the same number of components and by Whitney's Theorem [5], or see pp. 248 of [4] $G$ and $H$ have the same number of components of each isomorphism type with the possible exception of triangles and 3-pointed stars.

If for each $e \in E(G), e$ is from a triangle component of $G$ if and only if $\tau(e)$ is from a triangle component of $H$, then $G \cong H$ since they would have the same number of triangle components. If there is some $e \in E_{-}(G)$ such that $e$ is from a triangle component but $\tau(e)$ is not then $\tau(e)$ must be from a 3-pointed star component of $H$. But then $G^{e} \not H^{\tau(e)}$ since the latter has one more component than the former. (Removing $\tau(e)$ from the star leaves a path of length two and an isolated vertex.) One gets the same contradiction if $e$ is not from a triangle component while $\tau(e)$ is.

The proof that the vertex problem for line graphs is valid if the edge problem is valid is omitted because of its similarity to the above proof.

CoROLLARY. If $G$ is disconnected then $G$ can be reconstructed from the collection $\left\{G^{e}\right\}_{e \in E(G) \text {. }}$

Proof. $L(G)$ can be constructed from the collection $(L(G))_{e}$ by [2] since $L(G)$ is disconnected.

It should be pointed out that one can decide from the $G^{e}$ if $G$ is connected or not. This follows from the observation that $G$ is connected if and only if either $G^{e}$ is connected for some $e \in E(G), G^{e}$ is a forest with exactly two trees for all $e \in E(G)$ and for some $e \in E(G)$ neither component of $G^{e}$ is an isolated vertex, or else $G^{e}$ is a star plus an isolated vertex for each $e \in E(G)$.

\section{REFERENCES}

1. F. Harary and E. Palmer, The reconstruction of a tree from its maximal subtrees, Canad. J. Math. 18 (1966), 803-811. 
2. F. Harary, "On the reconstruction of a graph from a collection of subgraphs," pp. 47-52 in Symposium on the theory of graphs and its applications (Prague, 1964), Publ. House. Czech. Acad. Sci., Academic Press, New York, 1964.

3. P. J. Kelly, A congruence theorem for trees, Pacific J. Math. 7 (1957), 961-968.

4. Oystein Ore, Theory of graphs, Amer. Math. Soc. Colloq. Publ., Vol. 38, Amer. Math. Soc., Providence, R.I., 1962.

5. H. Whitney, Congruent graphs and the connectivity of graphs, Amer. J. Math. 54 (1932), 150-168.

VANDERBILT UNIVERSITY 